\title{
The Evaluation Index System and Model of Engineering Quality for Engineering Students \\ Xin-liang CAO ${ }^{1, a,{ }^{*}, \text { Hong-xia YANG }}{ }^{2, b}$ and Jian-xin $\mathrm{LI}^{1, \mathrm{c}}$ \\ 'School of Phy. and Elec. Infor., Yan'an University, Shaanxi Yan'an, China \\ ${ }^{2}$ School of Architectural Engineering, Yan'an University, Shaanxi Yan'an, China \\ aCaoxinliang874@163.com, byanghongxia72@126.com, cyaljx@126.com
}

Keywords: Engineering quality, Index system, Evaluation model

\begin{abstract}
In order to meet China Engineering Education Accreditation, and comprehensively improve the quality of engineering students, the tower Index System is explored. And Engineering Students' engineering Qualities and the appropriate evaluation model is established based on AHP. As an example by electronic information undergraduates, an engineering quality evaluation system is included and the quality evaluation method is studied. This work is to provide methods and reference for training quality engineering on quality self-assessment evaluation to schools.
\end{abstract}

\section{工科大学生工程素质评价指标体系与评价模型}

\author{
曹新亮 $^{1, a, *}$, 杨红霞 ${ }^{2, b}$, 李建新 ${ }^{1, c}$ \\ ${ }^{1}$ 延安大学物理与电子信息学院, 陕西延安, 中国 \\ 2 延安大学建筑工程学院, 陕西延安, 中国 \\ aCaoxinliang874@163.com, byanghongxia72@126.com, cyaljx@126.com
}

关键词:工程素质; 指标体系; 评价模型

摘 要: 为适应工程教育认证要求、全面提高工科大学生素质, 探索的工科大学生工程素质的 塔式指标体系, 依据层次分析法建立相应的评价模型。以电子信息类本科生为例, 列举出工 程素质评价指标体系、研究了一套评价方法, 为学校对工程素质培养质量自评提供评价方法 和参考依据。

\section{1. 引言}

中国工程教育认证体系于 2016 年 6 月正式加入 “华盛顿条约”，具备高素质的技术人才 也将成为世界公认的工程人才。

目前, 许多工科院校依据国际 CDIO 工程教育能力大纲与 12 条标准 ${ }^{[1]}$ 进行教学模式改革, 在教学实践把重心放在对学生的实践能力培养和职业能力的养成上 ${ }^{[2,3]}$ 。但是, 对将要毕业的 学生所具备工程素质的程度和素质教育效果还缺乏较系统的自评体系。文章针对电子信息类 专业学生应具备的工程素质要素进行分析, 依据定性与定量相结合的评价理论归纳建立工程 素质塔式评价指标体系和一种层次化评价模型, 为大学生工程素质评价提供科学依据。 


\section{2. 塔式评价指标体系}

依据中国工程教育认证通用标准的 对毕业要求 ${ }^{[4]}$ 和电子信息行业对工程素 质的要求, 并按照有利于促进学生的知 识、能力和素质一体化成长的要求为准 则, 把联合国教科文组织关于 21 世纪教 育中 “学习知识 (学科知识)、学会做人、 学会做事、学会共处” 四大支柱包含其 中, 突出能力 (创新能力、创业能力、 组织能力、团队能力、交流与写作能力） 形成的权重, 构成电子信息类大学生工 程素质塔式评价指标体系，如图 1。

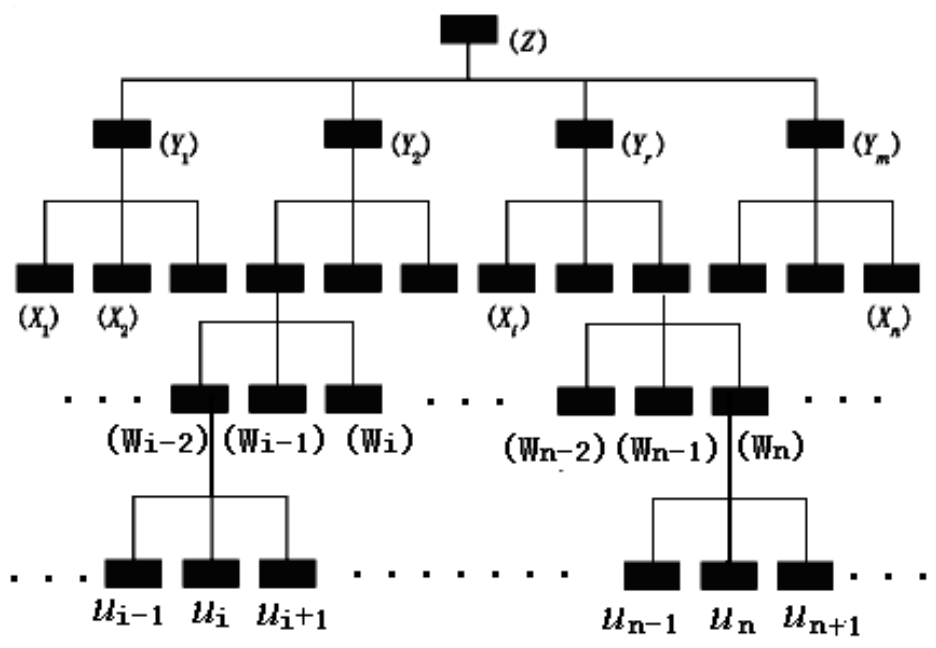
图 1 塔式评价指标体系

其中, 目标层 $(\mathrm{Z})$ 为工程素质培养效果评价结论; 准则层 $(\mathrm{Y})$ 包括知识掌握程度、专业能 力、职业素养三个方面; 在指标层 (X) 中分别涉及到数理基础、电路知识、工程知识等八 类指标; 子指标层 (W) 是对指标层内涵的割裂与细化, 在子指标层中包含有如对专业知识 割裂为电路知识、通信知识、计算机应用、软件知识, 对数理基础细化为建模、解算等十六 类子项; 要素层 $(u)$ 涉及到某些子指标层所含的具体要素共二十六种，如图 2。

\section{3. 工程素质评价原理及方法}

\section{1 评价原理}

工程素质评价要解决单指标评价和多指标评价结果的综合这两个主要问题。在对多指标 评价结果的综合过程中，可以采用层次分析法 ${ }^{[6]}$ 确定各指标对目标的影响权重。对事物定性 的描述称为属性, 属性综合评价系统可分为单指标属性测度、多指标属性测度和识别分析三 个子系统。单指标属性测度分析子系统是按照指标值的大小和评价类的关系确定属性测度函 数, 根据各指标的特征值计算其属性测度值; 多指标综合属性测度分析子系统是由单指标属 性测度经加权求和得到综合属性测度; 识别分析子系统是根据第二个子系统输出的结果给出 识别准则, 用以识别工程素质属于哪一个评价类, 对有序评价类, 需采用置信度准则。

\section{2 定性与定量相结合的评价方法}

\subsection{1 定性判断}

把准则层 $(\mathrm{Y})$ 、指标层 $(\mathrm{X})$ 、子指标层 $(\mathrm{W})$ 、要素层 $(u)$ 的各层所含要素重要性定 性评判结果分为: 微小重要、稍为重要、更为重要、明显重要、十分重要、强烈重要、更强 列重要、极端重要九个等级, 由专家分别对各层次要素重要性作定性判断。

\subsection{2 定性向定量判断过渡一一构造判断矩阵}

依据专家对要素的定性判断, 按重要性等级排序、并进行两两比较, 用其倒数的标度将 各要素的重要性进行判断定量化, 构造判断矩阵。方法为: 若要素 $i$ 比要素 $j$ 更为重要, 则 $a_{i j}=4 、 a_{j i}=1 / 4$; 要素 $i$ 与要素 $j$ 同等重要, 则 $a_{i i}=a_{j j}=1$; 若要素 $i$ 处于稍微重要而要素 $j$ 处 于明显重要, 则 $a_{i j}=1 / 3 、 a_{j i}=3$ 等。该层指标要素个数就是该层判断矩阵阶数。 


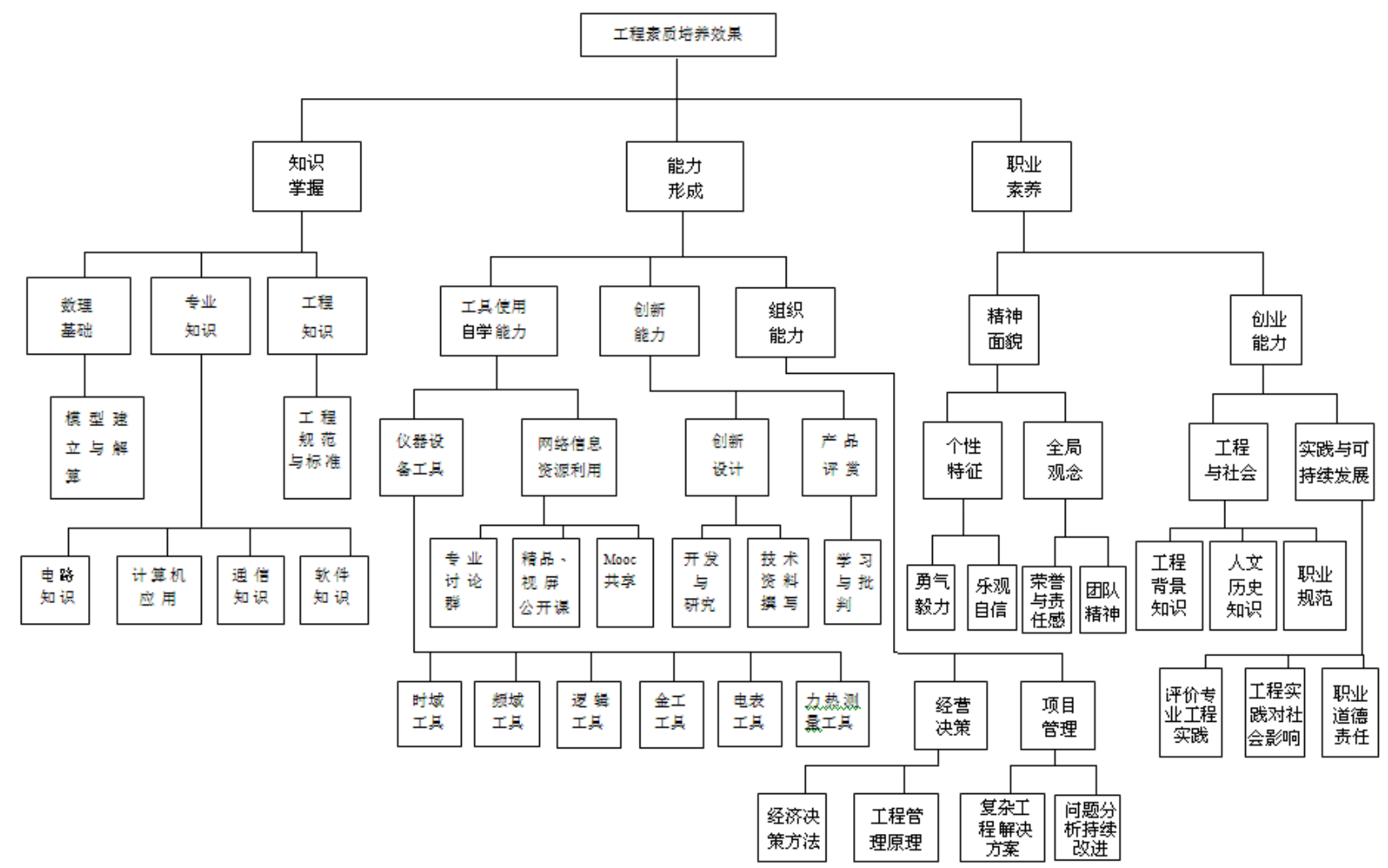

图 2 工程素质评价塔式指标体系

计算判断矩阵的最大特征值和特征向量, 并经一致性检验后, 确定该层各指标的权重。 以准则层为例, 根据专家定性判断: “职业素养”明显重要、“知识掌握”十分重要、“专 业能力” 形成极端重要。则判断矩阵构造为: $\left[\begin{array}{ccc}1 & 2 & 5 \\ \frac{1}{2} & 1 & 4 \\ \frac{1}{5} & \frac{1}{4} & 1\end{array}\right]$, 解其权值分别为: $0.095 、 0.335$ 、 0.570 。 此即职业素养、知识掌握、专业能力对目标层的权重。

同理，可求得以下各层对上一层权值，进而可计算得到不相邻低层次对高层次的权值。 以图1中前三层指标体系为例, 可用层次分析法确定准则层 $Y$ 的 $r$ 准则相对于目标层Z的重要性 权值 $\omega Y_{r}$ 和指标层 $\mathrm{X}$ 的 $\mathrm{i}$ 指标相对于准则层 $\mathrm{r}$ 准则的单排序权值 $\omega X_{i r}$, 利用合成权重计算法可求出 指标层的 $\mathrm{i}$ 指标相对于目标层的权值 $\omega_{i}$ 为:

$$
\omega_{i}=\sum_{r=1}^{m}\left(\omega Y_{r} \cdot \omega X_{i r}\right) \quad(i=1,2, \cdots, n)
$$

\section{2 .3 单指标属性测度分析}

评价体系中的指标有定量指标和定性指标两类。定量指标的特征值可根据具体参数的数 值确定; 定性指标可在统一标准的前提下由判断矩阵获得特征值。工程素质某单指标要素评 价若分为优、良、一般三个等级, 即对应的评价集为 $\left(V_{1} 、 V_{2} 、 V_{2}\right)$ 。当第 $\mathrm{i}$ 个指标的特征量 为 $t_{i}$ 时, $t_{i}$ 属于第 $k$ 个评价类, 可表示为 $t_{i} \in V_{k}(1 \leq k<3)$, 它的属性测度:

$$
\mu_{i k}=\mu\left(t_{i} \in V_{k}\right) \quad\left(\mu_{i k} \text { 满足: } \mu_{i k} \geq 0, \sum_{k=1}^{3} \mu_{i k}=1\right)
$$

\subsection{4 多指标综合属性测度分析}

由层次分析法求出各要素的权重值 $\omega_{i}$, 由单指标属性测度函数求出各指标的属性测度 $\mu_{k}$, 采用加权求和法可得工程素质评价的多指标综合属性测度:

$$
\mu_{k}=\sum_{k=1}^{n} \omega_{i k} \mu_{i k} \quad(1 \leq k \leq 3)
$$




\section{2 .5 识别分析}

对有序评价类 $(\mathrm{v} 1, \mathrm{v} 2, \mathrm{v} 3)$, 需采用置信度准则对综合属性测度 $\mu k$ 进行识别。置信度 $\lambda$ 一般在 0.6 0.7之间取值, 若 $k_{\min }=\min \left\{k \mid \sum_{e=1}^{k} \mu_{e} \geq \lambda, \quad 1 \leq k \leq 3\right\}$

则评价属 $V_{k}$ 类。对工程素质评价进行多指标综合量化评价的结论最终由（4）式给定。

以上（1）、（2）、（3）、（4）式体现了层次化指标体系由定性到定量、再到属类的 评价顺序和方法, 由此而获得了电子信息类工程素的层次分析法评价模型,

\section{致谢}

本文为 “陕西省高等教育教学改革研究项目基金”(13BY53); 陕西省高水平大学建设专 项资金(2015SXTS02)和延安大学教学改革重点项目（YDJGZD2016-03）的成果之一。

\section{References}

[1] GU Pei-hua, LU Xiao-hua, SHEN Min-fen (Compilation), The Outline and Standard of CDIO, Shantou University Press, in Guangdong of China, (In Chinese with English abstract),2008.

[2] DOU Fang-fang, LI Jia-min, The Problem-Based Interdisciplinary Learning: The Reform of Undergraduate Teaching in Colleges and Universities, Higher Education Exploration, issue.10, pp.82-86,2015.

[3] LI Yong-shan, Study on the Construction and Implementation of the Curriculum System of College Students' Quality Education,China Higher Education, issue.11, pp. 53-55,2015.

[4] China Engineering Education Professional Certification Association, Engineering Education Certification Standard, China Higher Education Evaluation Center of Ministry of Education, 2015 Edition.

[5] ZHU Shou-xin, Yang Jie, Based on Quality Standard of Higher Maturity Evaluation Construction, Jiangsu Higher Education ,issue.10, pp.10-13,2015.

[6] FENG Li-xia,SHI-Shao-ting,DU Wen-ming, The model of teaching evaluation index based on AHP, Journal of Northwest Normal University(Natural Science), vol. 46, pp. 19-23, 2010. 